\title{
Image Processing for Virtual Histology of the Vestibular End-Organs
}

\author{
Hisaya Tanioka, $\mathrm{MD}, \mathrm{PhD}^{1}$, Kimitaka Kaga, $\mathrm{MD}, \mathrm{PhD}^{2}$ \\ ${ }^{1}$ Department of Radiology, Tanioka Clinic, 6-24-2 Honkomagome, Bunkyo-ku, Tokyo 113-0021, Japan. \\ ${ }^{2}$ National Institute of Sensory Organs, Tokyo Medical Center, Tokyo 152-8902, Japan
}

\begin{abstract}
The living anatomy of the vestibular end-organs is still unknown, complex, and studying them is a challenge for researchers in basic science and in clinical medicine. This main reason is the fact that we cannot observe the vestibular end-organs in the living subjects by any modern diagnostic facilities such as CT and MRI. We can make the three-dimensional virtual histology of the vestibular end-organs based on high-resolution computed tomography (HR-CT) of a human temporal bone with the use of the direct volume rendering. The three-dimensional virtual histology is a volume rendering of these structures of interest, which currently includes the bone, soft tissues and air spaces of the temporal bone; the maculae of utricle and saccule, otolith, ampullae, and the ossicles. The three-dimensional volume rendering imaging can be sliced open at any section and the appropriate raw radiological image superimposed on the cleavage plane. The images stack can be resected in any arbitrary plane. This virtual histological technique is a powerful investigating tool for studying the complex anatomy of the vestibular end-organs of living subjects. We can research morphological conditions of the vestibular end-organs of normal functional status and pathological conditions like a retinal camera.
\end{abstract}

Keywords-volume rendering; virtual histology; vestibular end-organs; CT.

\section{INTRODUCTION}

According to amazing progress of radiological imaging modalities has resulted in steady increase in volume and improvement in accuracy of the imaging information of the inner ear, morphological and biomedical researchers can observe images of the inner ear in the cadaver at a level of a light microscope by using micro-CT and micro-MRI. Even though, no any current facilities can detect the vestibular end-organs in the living inner ear. We can reconstruct the three-dimensional virtual histology of the vestibular end-organs in the membranous labyrinth using by a volume rendering. As a result, our knowledge of the living vestibular apparatus under normal and pathological conditions will became more complete in living subjects. For all researchers and physicians of vertigo, disequilibrium, and dizziness, it is the long standing dream to obtain histological images of the vestibular end-organs in living subjects under suffering diseases and balance functional testing. Our representative technique will be possible to study of living subjects for vestibular researches.

In this paper, the principles, methodology, interpretation, and applications of the three-dimensional virtual histology of the vestibular end-organs in the membranous labyrinth will be mentioned by compared with previous papers and anatomical books.

\section{MATERIALS AND METHODS}

Imaging data from 4 volunteers ( 2 men and 2 women, mean age 58) who visited Tanioka Clinic for their regular checkup were used to reconstruct high-resolution images of the temporal bone. These subjects had no known affliction of the temporal bone and no history of hearing problem, dizziness, or other symptoms related to vestibulocochlear systems and normal findings on both CT exam and physical exam by checkup. Informed consents were obtained from all participants, and our ethics committee approved the study protocol.

\section{A. CT Protocol}

All examinations were performed with a spiral CT scanner (ProSpeed AI; General Electric Systems, Milwaukee, Wis., USA). The section thickness was $1 \mathrm{~mm}$ and an interval of $0.5 \mathrm{~mm}$ overlapping. A pitch was 1.0. The axial images were reconstructed with a high-resolution algorithm in steps of $0.5 \mathrm{~mm}$ and a field of view (FOV) of $96 \times 96 \mathrm{~mm}$. By using a 512X512 matrix, the resulting voxel size was $0.50 \mathrm{X} 0.18 \mathrm{X} 0.18 \mathrm{~mm}$.

\section{B. Anatomy and Imaging Principles of the Vestibular end-organs}

The vestibular labyrinth consists of the utricle and saccule, the three ampullae, the three semicircular ducts, the endolymphatic duct and sac, and these systems of nerve fibers. As all functional tissues of the inner ear are within the membranous labyrinth embedded in the endolymph surrounded by the perilymph, in view of the matter, we make to reconstruct images of soft tissues within the fluid capsule such as pulmonary nodules in the bronchus like an air tube surrounded by the air of lung. That is to say, the bronchial tube replaces with the vestibular membrane, the air replaces by the endo and perilymphatic fluids, and the bronchial tumor was changed by the soft tissues in the membranous labyrinth. So far, direct volume rendering can be applied to visualization of the membranous labyrinth such as pulmonary nodules [1].

\section{Postprocessing}

After transferring the image data to a CT workstation (GE Medical Systems), three-dimensional visualization based on interactive direct volume rendering was made using General Electric Advantage Navigator software; Volume Rendering Ver.2. The direct volume rendering 
considers some of the image data, so roughly simple explicit segmentation prior to the visualization process was required using cut-plane. And, next, we select voxel values of CT data. There are two images are reconstructed by choosing the item of 'show voxel values' or 'do not show voxel values'. When the voxel values are off, the image will be shaded using the average of the voxel values as weighted by their contribution to the pixel values. As a result of this software, both color and opacity values were adjusted interactively to delineate all structures related to the membranous labyrinth in real time. After an appropriate setting using the front cut for optimal delineation of the target structures is defined on axial, coronal and sagittal projection, the color and opacity table can be stored and used for further studies. Owing to the simple acceleration of the visualization process, the whole procedure was performed in less than 1 minute. The standardized user ensured intuitive manipulation of any object in real time. The software allows both distance measurements and volume directly within the three-dimensional scene. The main target structures were (a) the utricular macula, (b) the saccular macula, (c) the otolith, (d) the ampullae, (e) the round window, and (f) the oval window. Digital process in generating virtual histology is shown by Fig. 1.

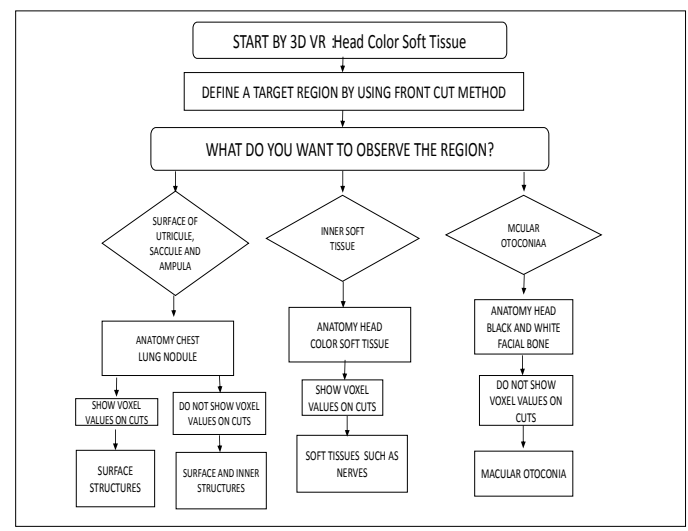

Figure 1. Digital process in generating virtual histology

\section{RESULTS}

In the 8 normal ears of the temporal bone, high-quality three-dimensional visualization of the main target structure was possible. For optimal delineation of the vestibular end-organs, the structures surrounding the otic capsule were suppressed from visualization with all planes. Our three-dimensional images of the vestibular end-organs allowed high-contrast differential anatomic structures and these identifications of each structures as the membranous semicircular ducts, the saccule and utricle (Fig. 2, 3 and 4). Our delineated all images agreed with those of the schematic diagram of structure of the membranous labyrinth represented by anatomic books (Fig. 6) [2, 3, 4, 5].

\section{A. Location and shape.}

Utricle. At the level of the lower border of the lateral semicircular duct, the major plane of the utricle lies parallel to the plane of the lateral duct (Fig. 2). The utricle originates in the elliptical recess in the anterosuperior vestibule and narrows as it extends posterinferiorly. Thus, from the superior and inferior aspects the utricle looks like an acorn or a shield (Fig. 2 and 3). The otolith of the utricular macula showed an oblong heart shape resembled to previous papers (Fig. 5) $[2,3,4,5]$.

Saccule. The saccule is located in the spherical recess in the anteromedial part of the vestibule at the level of the oval window or just below the lateral semicircular canal plane (Fig. 2). In anterolateral and inferiormedial views, the saccule looks like an elongated kidney bean (Fig. 2, 3 and 4) $[2,3,4,5]$.

Ampulla. The ampullae showed fiddle-shaped (Fig. 2, 3 and 4). Fig. 4 represented the internal anatomical shape of the soft tissues in the superior and lateral ampullae [3].

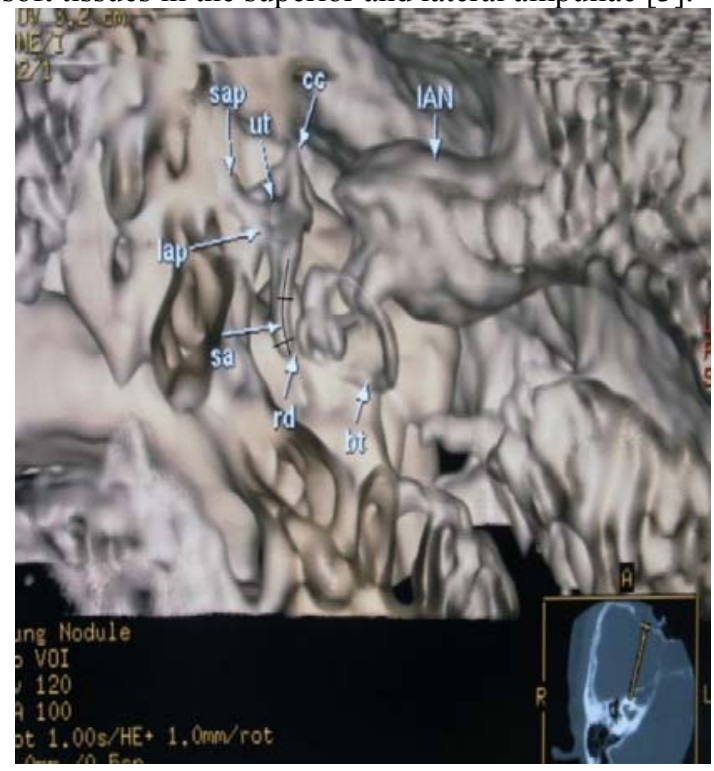

Figure 2. This anterosuperior image was obtained by the method of surface structures. The length of the saccule was along the line shown in this figure. The saccule clearly lies in the three-dimensional plane.

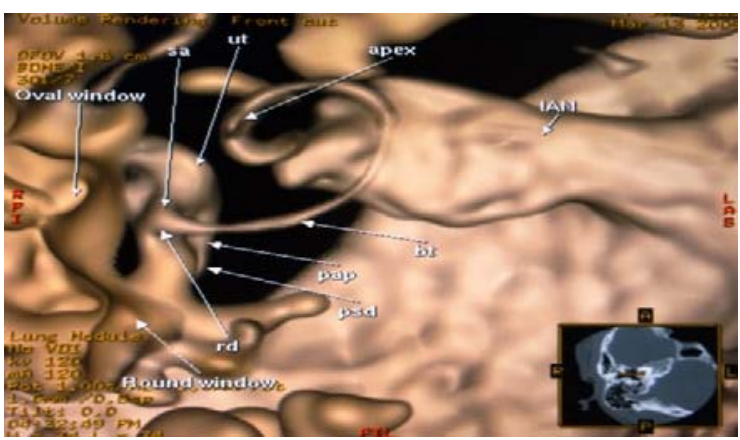

Figure 3. The inferiormedial aspect of the vestibule, semicircular duct and cochlear duct. The saccule is located in the anteromedial part of the vestibule. This image was made by the method of surface structures. 


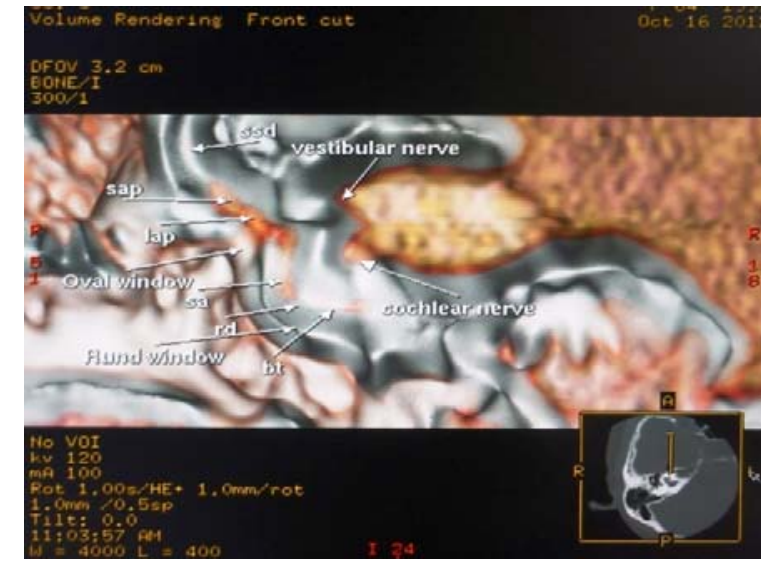

Figure 4. Anterior view of the soft tissue structures of the inner ear obtained by the method of soft tissues such as nerves. The superior and lateral ampullae were seen in this figure. The internal anatomy of the soft tissues could be seen on this figure.

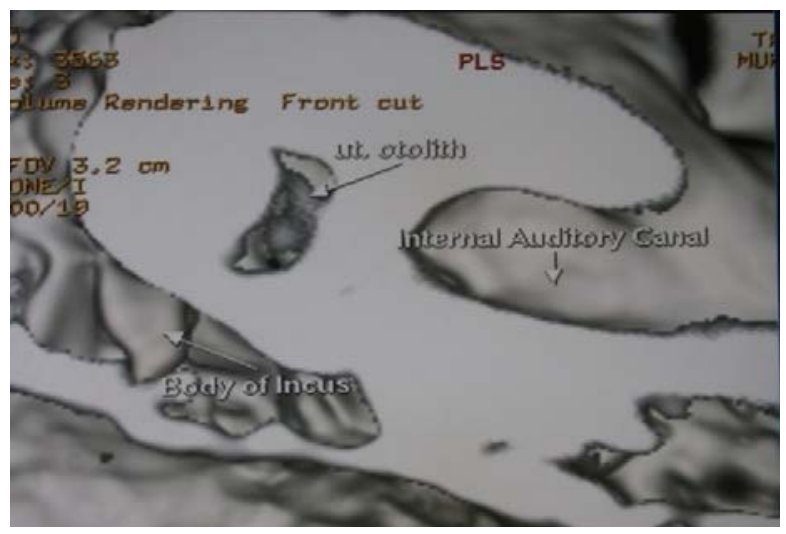

Figure 5. Superior view of the utricle obtained by the method of macular otoconia. The otolith of the utricular macula can be visible shaped like an oblong heart figure (ut. otolith)

\section{Schema of the Inner Ear}

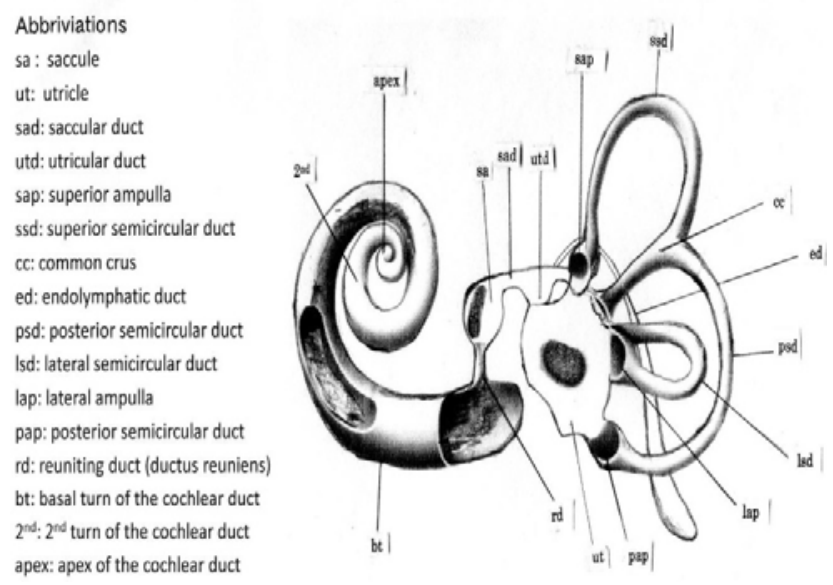

Figure 6. Schema of the membranous labyrinth in the inner ear. (Draw by Tanioka $\mathrm{H}$ )

\section{DISCUSSION}

The three-dimensional reconstructed imaging method of the vestibular end-organs is a new approach to interactive volume rendering analysis of the vestibule. Major advantages of this technique are that it is reliability performed with standard spiral CT examinations and dose not requires complicated explicit segmentation of the image data. Moreover, the whole procedure is performed within a very short time; it is suitable for application in routine clinical work such as a retinal camera.

For the anatomical research of the vestibular end-organs in the inner ear, the standard procedure has been histopathological sectioning microtome following chemical fixation and deossification of the temporal bone. But, our virtual histological technique does not require such a troublesome work, and more, we can study the vestibular end-organs in living subjects in real time. So, this method will change the anatomical study of the vestibular end-organs in the inner ear.

\section{A. Problems}

A major problem in the three-dimensional reconstructed imaging of the vestibular end-organs obtained by high-resolution computed tomography (HR-CT) is the radiation exposure. In this study, the dose of radiation exposure was that the range of DLP (Dose Length Projection) in projection was $45 \mathrm{mGy} / \mathrm{cm}$ to $55 \mathrm{mGy} / \mathrm{cm}$. This value of radiation exposure was allowed permissible range for the exam [6].

\section{B. Clinical use of the three-dimensional reconstructed imaging of the vestibular end-organs}

In this study, we demonstrated for usefulness of the three-dimensional reconstructed imaging of the vestibular end-organs as virtual histology in case of normal subjects. By using the interactive direct volume rendering of our method, the relationship between the structures of the vestibular end-organs in the inner ear is understood easily in living subjects. Our creative method enables to understand the status of the vestibular end-organs under the condition of normal and abnormal level. These facts imply that we can know conditions of the otolith systems, ampullae, utricle and saccule without a dissection of the temporal bone in the situation of vertigo, disequilibrium, dizziness, and aging changes. And, as we can know morphological features of the otolith systems in living subjects, this method will contribute to elucidate the special function of the otolithic organs in the general vestibular capacity of equilibrium maintenance.

Although we believe this method is useful for diagnosis and treatments such as Meniere's disease, benign paroxysmal positional vertigo (BPPV), dizziness and aging changes; we must study to connect this creative method to the physiology of these diseases and their clinical courses.

\section{REFERENCES}

[1] Remy-Jardin M, Remy J, Artaud D, Fribourg M, Duhamel A. Volume rendering of the tracheobronchial tree: clinical evaluation of bronchographic images. Radiology, 208, pp. 761-770, 1998. 
[2] Takagi A, Sando I. Computer-aided three-dimensional reconstruction and measurement of the vestibular end-organs. Otolaryngol Head Neck Surg., 88, pp. 195-202, 1988.

[3] Beck C, Bader J. Ein Beitrag zur feineren Anatomie des menschlichen Innenohres. Zusammengefa $\beta \mathrm{t}$ dargestellt in einem neuen Labyrinthmodell. (A contribution to fine anatomy of human inner ears, with structure of a new labyrinth model) Archiv Ohren-usw. Heilk. u. Z. Hals-usw. Heilk., 181, pp. 245-267, 1972.
[4] Rosenhall U. Vestibular macula mapping in man. Ann Otol Rhinol Laryngol., 81, pp. 339-351, 1972.

[5] Corvera J, Hallpike CS, Schuster EHJ. A new method for the anatomical reconstruction of the human macular planes. Acta Otolaryngol., 49, pp. 4-16, 1958.

[6] European guideline on quality criteria for computed tomography (EUR 16262) 